\title{
Mild endothelial dysfunction in Sirt3 knockout mice fed a high- cholesterol diet: protective role of a novel C/EBP- $\beta$-dependent feedback regulation of SOD2
}

\author{
Stephan Winnik ${ }^{1,2}$ - Daniel S. Gaul ${ }^{2,6}$ - Giovanni Siciliani ${ }^{2}$ - Christine Lohmann ${ }^{2}$ • \\ Lisa Pasterk $^{2,3}$ - Natacha Calatayud ${ }^{2}$ - Julien Weber ${ }^{2}$ - Urs Eriksson ${ }^{2,4}$. \\ Johan Auwerx ${ }^{5}$ - Lambertus J. van Tits ${ }^{2}$ - Thomas F. Lüscher ${ }^{1,2,6}$. \\ Christian M. Matter ${ }^{1,2,6}$
}

Received: 22 July 2015/Accepted: 30 March 2016/Published online: 12 April 2016

(c) The Author(s) 2016. This article is published with open access at Springerlink.com

\begin{abstract}
Sirtuin 3 (Sirt3) is an $\mathrm{NAD}^{+}$-dependent mitochondrial deacetylase associated with superoxide dismutase 2 (SOD2)-mediated protection from oxidative stress. We have reported accelerated weight gain and impaired metabolic flexibility in atherosclerotic Sirt $^{-1-}$ mice. Oxidative stress is a hallmark of endothelial dysfunction. Yet, the role of Sirt3 in this context remains unknown. Thus, we aimed to unravel the effects of endogenous Sirt3 on endothelial function and oxidative stress. Knockdown of Sirt3 in human aortic endothelial cells (HAEC) increased intracellular mitochondrial superoxide accumulation, as
\end{abstract}

S. Winnik and D. S. Gaul contributed equally.

Electronic supplementary material The online version of this article (doi:10.1007/s00395-016-0552-7) contains supplementary material, which is available to authorized users.

Stephan Winnik

stephan.winnik@usz.ch

Christian M. Matter

christian.matter@usz.ch

1 Department of Cardiology, University Heart Center Zurich, University Hospital Zurich, Raemistr. 100, 8091 Zurich, Switzerland

2 Center for Molecular Cardiology, University of Zurich, Schlieren, Switzerland

3 Institute of Experimental and Clinical Pharmacology, Medical University of Graz, Graz, Austria

4 Division of Cardiology, Department of Medicine, GZO Regional Health Center Wetzikon, Wetzikon, Switzerland

5 Laboratory of Integrative Systems Physiology, School of Life Science, Ecole Polytechnique Fédérale de Lausanne, Lausanne, Switzerland

6 Zurich Center for Integrative Human Physiology, University of Zurich, Zurich, Switzerland assessed by electron spin resonance spectroscopy and fluorescence imaging. Endothelium-dependent relaxation of aortic rings from Sirt $3^{-/}$mice exposed to a normal diet did not differ from wild-type controls. However, following 12 weeks of high-cholesterol diet and increasing oxidative stress, endothelial function of Sirt $3^{-/-}$mice was mildly impaired compared with wild-type controls. Relaxation was restored upon enhanced superoxide scavenging using pegylated superoxide dismutase. Knockdown of Sirt3 in cultured HAEC diminished SOD2 specific activity, which was compensated for by a CCAAT/enhancer binding protein beta (C/EBP- $\beta)$-dependent transcriptional induction of SOD2. Abrogation of this feedback regulation by simultaneous knockdown of $C / E B P-\beta$ and Sirt3 exacerbated mitochondrial superoxide accumulation and culminated into endothelial cell death upon prolonged culture. Taken together, Sirt3 deficiency induces a mild, superoxide-dependent endothelial dysfunction in mice fed a high-cholesterol diet. In cultured endothelial cells, a novel C/EBP- $\beta$-dependent rescue mechanism maintains net SOD2 activity upon transient knockdown of Sirt3.

Keywords Sirt 3 - Oxidative stress - SOD2 - C/EBP- $\beta$. Endothelial function

\section{Introduction}

Sirtuin 3 (Sirt3) is a mitochondrial deacetylase that regulates mitochondrial oxidative metabolism and detoxification from oxygen radicals $[1,3,44,58]$. It is the third out of seven mammalian homologues of the so-called class III histone deacetylases (HDACs). They share a conserved catalytic binding motif for the oxidized form of nicotinamide adenine dinucleotide $\left(\mathrm{NAD}^{+}\right)$, defining them as 
class III HDACs and concentrating their activity to times of low cellular energy levels [7, 9, 14, 19, 58]. Since its first description in 1935 , caloric restriction is the only intervention that has been shown to prolong lifespan and maintain mammalian health [25, 32, 47]. Mammalian sirtuins provide protective effects in a variety of age-related pathologies, thus promoting healthy aging [7, 13, 23, 34]. Sirt3 is one out of two sirtuins that have been associated with human longevity and health span [4, 15, 46]. Cardiovascular diseases, including myocardial infarction and stroke, are the leading global cause for age-related morbidity and mortality, accounting for 17.3 million deaths a year, with an estimated rise to $>23.6$ million by 2030 [2, 26].

Recently, Sirt3 deficiency has been reported to accelerate the development of the metabolic syndrome, a cluster of hallmark risk factors for cardiovascular disease [18]. Although we did not observe an atherosclerotic phenotype in low-density lipoprotein receptor knockout mice lacking Sirt3, its loss was associated with accelerated weight gain, impaired metabolic adaptation and increased levels of systemic oxidative stress [55].

Excess endothelial reactive oxygen species (ROS), subsequent mitochondrial DNA damage and progressive respiratory chain dysfunction are essential in the development of endothelial dysfunction, an early event in and an independent predictor of cardiovascular disease [17, 28, 29, 33, 36, 40, 41, 48]. In numerous settings including embryonic development, age-related hearing loss, neuronal injury, and cardiac hypertrophy, Sirt3 has been shown to protect from oxidative stress $[10,22,49,51]$. In the majority of settings, Sirt3 augmented superoxide scavenging by enhancing superoxide dismutase 2 (SOD2) and/ or catalase (cat) activity [42, 51, 53]. Whether increased SOD2 activity is mediated by direct deacetylation or transcriptional regulation remains controversial [51, 53]. Moreover, Sirt3 activates isocitrate dehydrogenase 2 (IDH2), which drives the tricarboxylic acid (TCA) cycle and is a major donor of NADPH, an essential cofactor for both glutathione regeneration and endothelial nitric oxide synthase (eNOS)-mediated NO formation [31, 43, 44, 59]. Hitherto, only few data exist on the role of Sirt3 in arterial endothelial cells [35]. No data are available on its effects on endothelium-dependent vasodilation.

In contrast to the constitutive expression of the cytosolic and extracellular isoforms SOD1 and SOD3, the inducible expression of SOD2 allows the response to varying levels of intracellular oxidative stress. The acetylation-dependent transcription factor CCAAT/enhancer binding protein beta $(\mathrm{C} / \mathrm{EBP}-\beta)$ has been shown to bind an intronic TNF-responsive element of SOD2 and thereby facilitate SOD2 gene transcription in response to various stimuli associated with increased levels of intracellular $\operatorname{ROS}[6,21,30]$.
Our goal was to unravel the effects of endothelial Sirt3 on oxidative stress and endothelial function, and to investigate the underlying mechanisms in human aortic endothelial cells. To enhance oxidative stress in aortae, we exposed mice to a high-cholesterol diet [37]. In the current work, we uncovered a C/EBP- $\beta$-dependent induction of SOD2 expression as rescue mechanism for the Sirt3-dependent loss of SOD2 activity, an interaction, that until to date remained unknown.

\section{Methods}

\section{Mice}

Mice were housed in a temperature-controlled facility with a 12-h light/dark cycle and free access to chow and water. All animal studies have been approved by the appropriate ethics committee and have therefore been performed in accordance with the ethical standards laid down in the 1964 Declaration of Helsinki and its later amendments. Mice with a germline Sirt3 deletion were generated as described. $[12,55]$ Congenic C57BL6/J Sirt $3^{-/}$mice were generated through nine generations of backcrosses with C57BL6/J mice. Eight-week-old male Sirt $3^{-/}$and wild-type mice were fed a $1.25 \%$ (w/w) cholesterol diet (research diets) for 12 weeks and subsequently killed for fasted (unless indicated otherwise) studies.

\section{Endothelial function}

Endothelium-dependent vasorelaxation was investigated as described [37, 56]. Briefly, aortae were explanted and aortic rings were obtained. Relaxation in response to acetylcholine $(\mathrm{ACh})$ or sodium nitroprusside (SNP) was assessed using isometric force transducers in organ chamber baths (Multimyograph, DMT). Maximal contraction was defined before initiating the experiment using potassium chloride $(\mathrm{KCl})$ in a concentration of $80 \mathrm{mM}$. Precontraction to a maximum of $70 \%$ maximal contraction was achieved using norepinephrine (NE) in a dose of $10^{-7} \mathrm{M}$. Dose-response curves were quantified comparing areas under the curves (AUC).

\section{Cell culture and transfection}

Human aortic endothelial cells (HAEC, Cambrex) from passage three to eight were grown to confluence at $5 \%$ $\mathrm{CO}_{2}$ and $37{ }^{\circ} \mathrm{C}$ in Endothelial Growth Medium 2 (Lonza) supplemented with $10 \%$ fetal calf serum. Transient knockdown was performed using Lipofectamine ${ }^{\circledR}$ Reagent (Life Technologies) for transfection of the following small interference RNA (siRNA): Sirt3 (5'-GCC CAA CGU 
CAC UCA CUA CUU TT-3'), C/EBP- $\beta$ (Trilencer-27 siRNA, OriGene), SOD2 (5'-AAU GCU ACA AUA GAG CAG CUU TT-3'), scrambled (5'-UUC UCC GAA CGU GGC ACG ATT-3'), Trilencer-27 Universal Scrambled Negative Control siRNA (SR30004, Origene), and Silencer Negative Control \#5 siRNA (AM4642, Ambion). Total siRNA amounts were kept equal among all experiments. Where two-stage transfections (double-knockdown of Sirt3 and $C / E B P-\beta$ ) were performed, all groups in the respective experiments were transfected twice. Knockdown efficiency was assessed using expression analyses on RNA- (quantitative PCR) and protein level (western blot).

\section{Expression analyses}

RNA isolation, reverse transcription and $\mathrm{SYBR}^{\circledR}$ greenbased (Applied Biosystems) quantitative PCR was carried according to standard protocols using a Quant Studio 7 Flex Real Time PCR thermocycler (Applied Biosystems) with the associated sequence detection system and software. Expression was calculated using the $\Delta \Delta C_{\mathrm{T}}$ method. Relative gene expression was normalized to $\beta$-actin (house-keeping gene). Western blot analyses of HAEC lysates were conducted according to standard protocols using the following specific antibodies:

anti-Sirt3 (rabbit monoclonal, Cell Signalling Technology), anti-C/EBP- $\beta$ [C19] (rabbit polyclonal, Santa Cruz Biotechnology), anti-SOD2 (rabbit polyclonal, Abcam), anti-catalase (mouse monoclonal, Sigma), anti-glutathion peroxidase 1 (rabbit polyclonal, Novus Biologicals), antitotal eNOS (mouse polyclonal, BD Transduction Laboratories), anti-eNOS (pThr495) (mouse, monoclonal, BD Transduction Laboratories), anti-eNOS (pSer1177) (mouse, monoclonal, BD Transduction laboratories), antixanthine oxidase (rabbit polyclonal, Biorbit), anti-NADPH oxidase subunit p22 $2^{\text {phox }}$ (rabbit polyclonal, Biorbit), and anti- $\beta$ actin (mouse monoclonal, Sigma-Aldrich). Specific signals were detected using species-specific secondary antibodies.

\section{Immunoprecipitation}

HAEC were cultured in $10 \mathrm{~cm}$ cell culture dishes, transfected as described above and lysed in $1 \mathrm{ml}$ radioimmunoprecipitation assay (RIPA) buffer. Samples were kept on ice throughout IP steps. The lysates were pre-cleared with $30 \mu \mathrm{l}$ washed Protein G Agarose beads (Millipore) and, after removal of the beads, incubated over night with suitable monoclonal antibodies for SOD2, C/EBP- $\beta$ or Sp1, respectively. $30 \mu \mathrm{l}$ of washed Protein G Agarose beads were added and the mixture was incubated for $4.5 \mathrm{~h}$ with agitation on the incubation wheel. Beads-antibody-antigen complexes were separated from the lysates by centrifugation and the pellet washed three times with RIPA buffer. After adding $30 \mu \mathrm{l}$ of $4 \times$ Laemmli buffer, the samples were incubated at $60{ }^{\circ} \mathrm{C}$ with shaking for $10 \mathrm{~min}$ and the supernatant resulting from subsequent centrifugation was analyzed by western blotting.

The following antibodies were used for immunoprecipitation and subsequent determination of the acetylation or nitrosylation status, respectively: anti-SOD2 [1E8] (mouse monoclonal, Abnova), anti-acetyl lysine (rabbit polyclonal, Chemicon), and anti-nitro tyrosine [HM.11] (mouse monoclonal, Abcam).

\section{Electron spin resonance spectroscopy}

Intracellular superoxide in HAEC was detected by electron spin resonance (ESR) spectroscopy using the superoxidespecific spin trap 1-hydroxy-3-methoxy-2,2,5,5-tetramethylpyorrolidine (CMH, Noxygen) as described [50, 57].

\section{Mitochondrial superoxide detection}

Mitochondrial superoxide generation was investigated based on the oxidation and fluorogenic nucleic acid binding of a mitochondrial- and superoxide-specific probe (MitoSOX ${ }^{\mathrm{TM}}$, Invitrogen). Cells were stained according to the manufacturer's protocol and fixed with $4 \%$ paraformaldehyde afterwards. Fluorescence was quantified using an Olympus BX51 microscope. Micrographs were quantified using ImageJ (NIH).

\section{Scavenging of mitochondrial superoxide}

HAEC were handled and transfected as described above. Upon transfection with siRNA the medium was supplemented with $1 \mu \mathrm{M}$ mitoTEMPO (Sigma). Medium was replaced with fresh EGM-2 medium containing $1 \mu \mathrm{M}$ mitoTEMPO once, before harvesting the lysates for expression analyses or staining the cells for fluorescence imaging.

\section{Superoxide dismutase 2 (SOD2) activity}

Mitochondrial fractions of HAEC were separated from whole cell lysates by centrifugation. Enzymatic activity of SOD2 in HAEC was assessed based on its capacity to dismutate superoxide radicals generated by xanthine oxidase under controlled conditions, using the Superoxide Dismutase Assay Kit (Cayman Chemical). Superoxide radicals were detected by colorimetric oxidation of tetrazolium salt to formazan dye. Any remaining activity of SOD1 and 3 was inhibited using potassium cyanide 
$(1 \mathrm{mM})$ according the manufacturer's instructions. Enzymatic activity was normalized to SOD2 protein expression.

\section{Nitric oxide production}

HAEC were seeded into a 96 well plate and transfected as described above. At time of the assay, cells were incubated with Krebs buffer containing $0.25 \mu \mathrm{M}$ 4,5-diaminofluorescein diacetate (DAF-2, Sigma) in presence or absence of $10 \mu \mathrm{M}$ of the unspecific nitric oxide synthase (NOS) inhibitor $\mathrm{L}-\mathrm{N}^{5}$-(1-Iminoethyl)ornithine hydrochloride (L-NIO, Sigma), or $0.25 \mu \mathrm{M}$ of the DAF-2 negative control 4-aminofluorescein diacetate (4-AF-DA, Merck Millipore), respectively, for $20 \mathrm{~min}$ at $37{ }^{\circ} \mathrm{C}$. Then L-arginine with or without calcium-ionophore (positive control) was added to the wells and fluorescence read at 490/525 nm (excitation/ emission) to set the baseline. After $30 \mathrm{~min}$ the fluorescence was measured again and the percentage of nitric oxide increase calculated.

\section{Statistics}

Metric variables were assessed for distribution using Kolmogorov-Smirnov tests. For $n<4$ non-parametric distribution was assumed. Different groups were compared using unpaired Student's $t$, Mann-Whitney, one-way ANOVA tests with Bonferroni multiple comparison post hoc tests or Kruskal-Wallis tests with Dunn's post hoc analyses, where applicable. $p$ values are two-sided. Significance was accepted for an alpha-error $<0.05$. Data are presented as mean \pm SEM, if not indicated otherwise. Statistical analyses were performed using GraphPad Prism 5 for Mac OS X (GraphPad Software).

\section{Results}

\section{Transient knockdown of Sirt3 increases endothelial superoxide levels}

siRNA-mediated transient knockdown of Sirt3 in human aortic endothelial cells (HAEC) reached an efficiency of $81 \%$ on RNA- and $87 \%$ on protein level (Fig. 1a). Intracellular superoxide levels were increased two-fold upon knockdown of Sirt3, as quantified using electron spin resonance spectroscopy (Fig. 1b). With Sirt3 being a mitochondrial deacetylase we used a mitochondrial- and superoxide-specific probe (MitoSOX ${ }^{\mathrm{TM}}$ ) to identify the cellular compartment of increased superoxide. Fluorescence imaging revealed a two-fold increase in mitochondrial superoxide levels, identifying mitochondria as the source of increased oxidative stress during transient knockdown of Sirt3 (Fig. 1c).
Loss of Sirt3 is associated with a mild superoxidedependent impairment of endothelial function

To assess the functional relevance of elevated endothelial superoxide levels in the absence of Sirt3, aortic rings of Sirt $3^{-1-}$ and wild-type mice were explanted and endothelium-dependent relaxation was quantified in organ chamber baths. Surprisingly, aortic relaxation of $\mathrm{Sirt}^{-/-}$mice in response to acetylcholine (ACh) was unaltered compared with wild-type controls (Fig. 2a). However, upon 12 weeks of high-cholesterol diet, known to increase oxidative stress [33], aortic relaxation of both genotypes was less sensitive to $\mathrm{ACh}$ at low dosages and showed an overall mild impairment in aortae of Sirt $^{-/-}$mice compared to wildtype controls (Fig. 2b). Scavenging endogenous superoxide by an excess of exogenous pegylated superoxide dismutase (PEG-SOD) improved the sensitivity to ACh of either genotype and abolished the impairment of aortic relaxation of high-cholesterol diet-fed $\mathrm{Sirt}^{-/-}$mice compared to wild-type controls (Fig. 2c). ACh-induced aortic relaxation in both genotypes could be prevented by preincubation with the endothelial nitric oxide synthase (eNOS) inhibitor L-nitroarginine methyl ester (L-NAME), indicating endothelial NO-dependency (Fig. 2d, S1C). Concomitantly, complete relaxation of aortae of both genotypes in response to the exogenous NO donor sodium nitroprusside (SNP) further underlined endothelium-derived NO-dependency (Fig S1A, B). Of note, there was no significant difference in body weight between wild-type and Sirt $3^{-/-}$ mice (Fig S2). These findings suggest a mild, superoxidedependent decline in aortic relaxation in the absence of Sirt3 upon a high-cholesterol diet.

\section{Endothelial SOD2-specific activity is diminished whereas SOD2 expression is increased following transient knockdown of Sirt3}

To unravel the mechanism underlying increased endothelial mitochondrial superoxide levels upon Sirt3 deficiency, we addressed SOD2-specific activity. Following transient knockdown of Sirt3 in HAEC, superoxide scavenging capacity of SOD2 was reduced by threefold compared with controls (Fig. 3a). Unexpectedly, expression levels of SOD2 were increased by 4.4 fold on RNA- and by 2.8 fold on protein level, respectively (Fig. 3c), thus compensating for the Sirt3-dependent loss of SOD2 specific activity; overall endothelial SOD2 activity, without normalizing to protein levels, was unchanged upon transient knockdown of Sirt3 (Fig. 3b). Decreased SOD2-specific activity was associated with a trend towards SOD2 hyperacetylation (Fig. 3d). SOD2 nitrosylation was unchanged upon knockdown of Sirt3 (Fig. 3e), suggesting a Sirt3-dependent, deacetylation-mediated activation of endothelial 
(A)
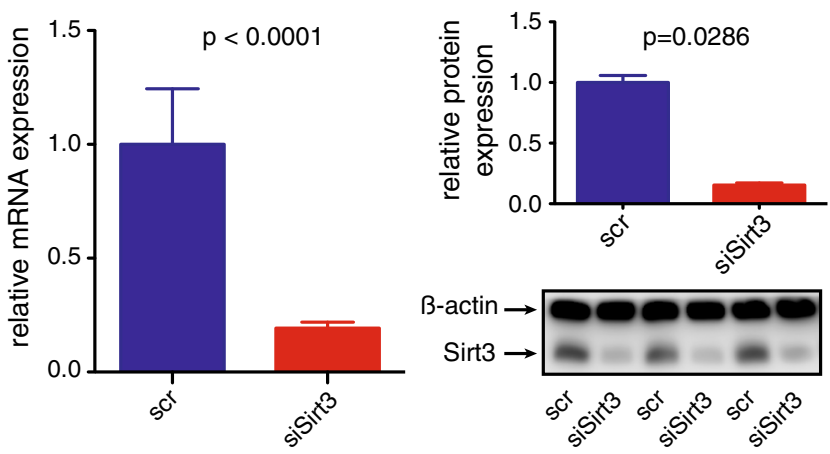

(B)

Cellular $\mathrm{O}_{2}^{-}$

(Electron spin resonance)

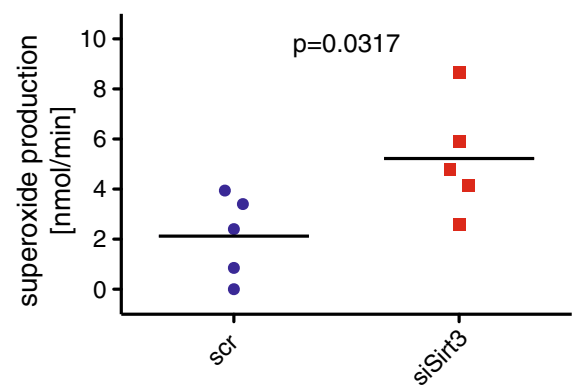

(C) Mitochondrial $\mathrm{O}_{2}^{-}$
(MitoSOX)

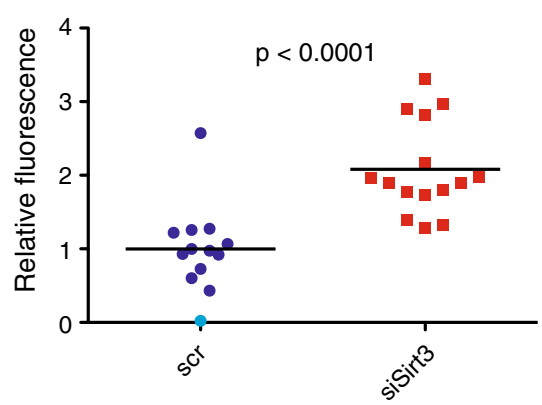

Fig. 1 Transient knockdown of Sirt3 increases endothelial superoxide levels. a Quantitative PCR of mRNA (left) and western blot analyses of protein (right) isolated from human aortic endothelial cells (HAEC) following siRNA-mediated knockdown of Sirt3. b Electron spin resonance (ESR) spectroscopy of live HAEC following siRNA-mediated knockdown of Sirt3 to quantify intracellular superoxide release. c Fluorescence imaging of HAEC following siRNA-mediated knockdown of Sirt3 and detection of mitochondrial

SOD2 under physiological conditions. Expression levels of other superoxide scavenging or decomposing enzymes, including SOD1, SOD3, catalase, thioredoxin 1, thioredoxin 2, thioredoxin-dependent peroxide reductase (PRDX3), and glutathione peroxidase were unaltered (Fig. 3f-h, Fig S3A-E). Accordingly, the expression level of endothelial superoxide-generating enzyme NADPH oxidase was unaffected by transient knockdown of Sirt3 (Fig S3F, G). Whereas no difference occurred in the cytosolic subunit $447^{\text {phox }}$ (Fig S3F), we observed a slight increase in the mRNA level of the membrane-bound subunit p22 $2^{\text {phox }}$, which did not translate into an increased protein level (Fig S3G).

\section{Nitric oxide generation is not affected by Sirt3 deficiency}

Assessment of endothelial nitric oxide synthase (eNOS) uncovered unaltered overall expression levels (Fig S4A) superoxide ( $r e d$ ) using MitoSOX ${ }^{\mathrm{TM}}$, a mitochondrial- and superoxidespecific probe; quantification on a per cell basis; representative micrographs show nuclei (blue) and mitochondrial superoxide (red, MitoSOX ${ }^{\mathrm{TM}}$ ); scale bars $20 \mu \mathrm{m}$. At least three independent experiments, each in biological triplicates, scr scrambled control, DAPI 4'6-diamidin-2-phenylindol, $\mathbf{b}$ and $\mathbf{c}$ show medians and single data points

and unchanged phosphorylation at Ser1177 (Fig S4B) upon Sirt3 knockdown. However, we observed a decreased phosphorylation of Thr 495 following transient knockdown of Sirt3 (Fig S4C), equivalent to an increased enzymatic activity. Together with an increased coupling of eNOS monomers (Fig S4D) this may be a compensatory effect secondary to an increased mitochondrial ROS accumulation upon Sirt3 deficiency. To assess the functional relevance of increased eNOS activity, nitric oxide (NO) generation was assessed in presence or absence of L-NIO, an unselective nitric oxide synthase inhibitor, using DAF-2 diacetate. L-NIO successfully reduced NO generation, however, no significant difference was observed upon transient knockdown of Sirt3 compared with controls, neither in presence nor in absence of L-NIO (Fig S4E). Thus, increased eNOS activity upon Sirt3 deficiency does not generate a detectable rise in NO. Nonetheless, increased eNOS coupling may contribute to counteract increased ROS levels upon Sirt3 deficiency. 
Fig. 2 Endothelial function is mildly impaired in Sirt $3^{-1-}$ mice in a superoxide-dependent manner. a Relaxation of aortic rings in response to increasing doses of acetylcholine (ACh) in Sirt $3^{-/-}$compared with wildtype mice fed a regular chow. b As in a following 12 weeks of a high-cholesterol diet. c As in $\mathbf{b}$, in presence of an excess of pegylated superoxide dismutase (PEG-SOD, $220 \mathrm{U} / \mathrm{ml}$ ), scavenging superoxide. $\mathbf{d}$ As in b, following preincubation with L-NAME (0.3 mM, $30 \mathrm{~min})$. $n=5-8$ mice per group, 4-7 rings per mouse, quantification of the areas under the curve (AUC), boxplots show interquartile ranges, whiskers indicate minima and maxima
(A) Relaxation to acetylcholine (ACh)

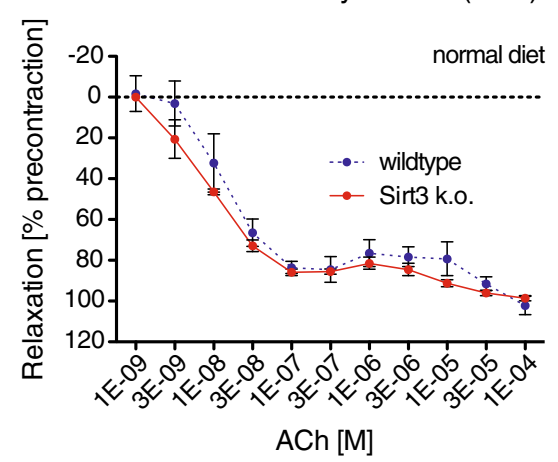

(B)

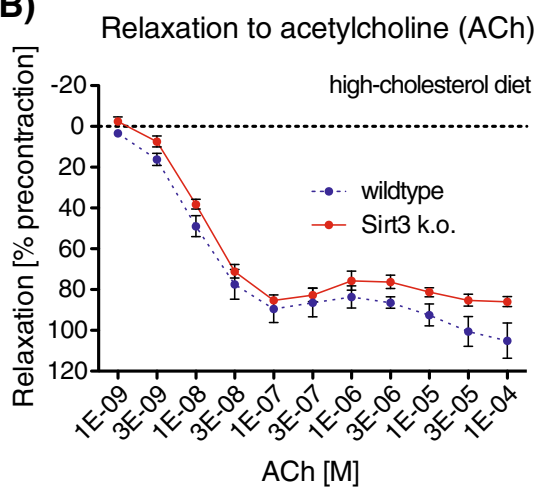

(C) Relaxation to acetylcholine (ACh) following PEG-SOD (220U/ml)

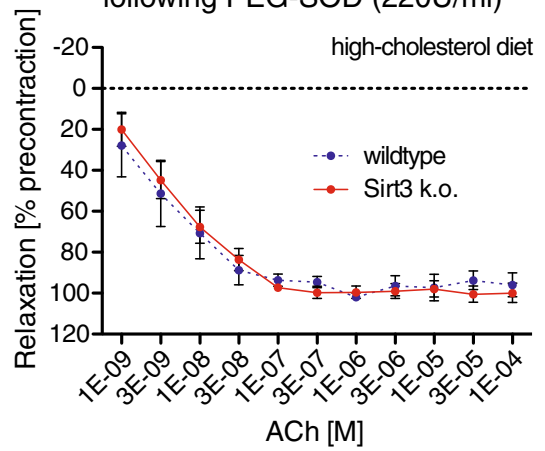

(D) Relaxation to acetylcholine (ACh) following L-NAME (0.3 mM)

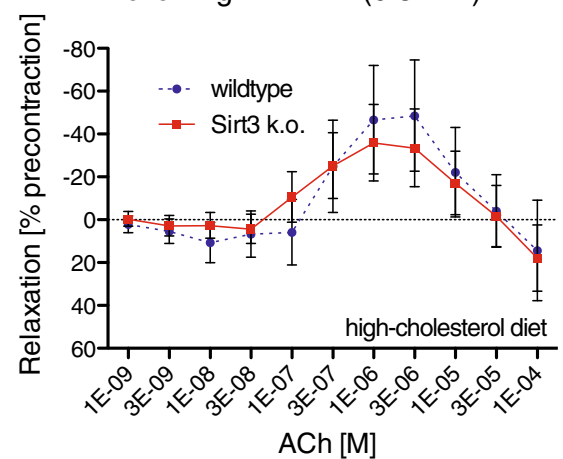

Area under the curve (AUC)

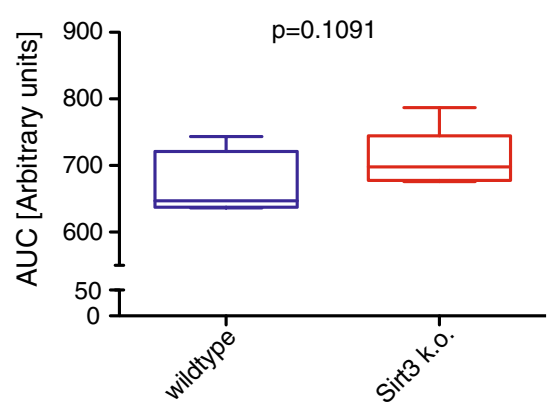

Area under the curve (AUC)

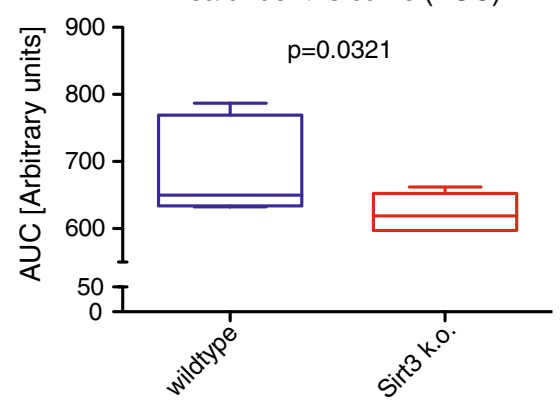

Area under the curve (AUC)

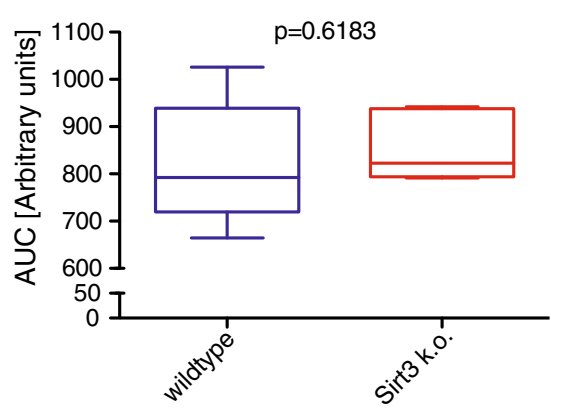

Area under the curve

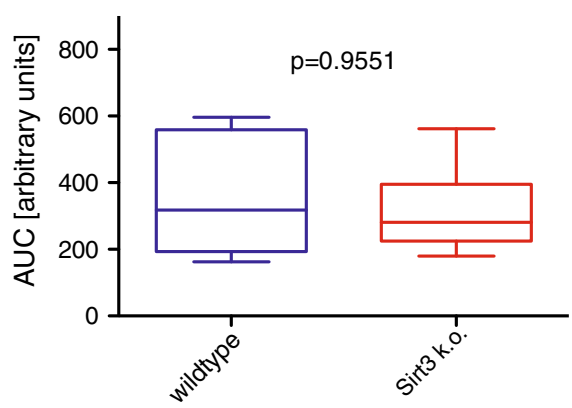


Fig. 3 Loss of Sirt3 decreases endothelial SOD2 activity and increases SOD2 expression without affecting other ROSscavenging or generating systems. a Superoxide dismutase 2 (SOD2) activity based on superoxide dismutating capacity in HAEC following siRNA-mediated knockdown of Sirt3; enzymatic activity was normalized to SOD2 protein expression; medians and single data points are shown. b Overall SOD2 activity, as in a without normalizing to protein content. c SOD2 mRNA (left) and protein $(r i g h t)$ expression in HAEC following siRNAmediated knockdown of Sirt3 using quantitative PCR and western blot, respectively. d Acetylation of SOD2, precipitated from HAEC following transient knockdown of Sirt3 using western blot analysis. e Nitrosylation of SOD2, precipitated from HAEC following transient knockdown of Sirt3 using western blot analysis. $\mathbf{f}-\mathbf{h}$ Expression analyses of $\mathbf{f}$ catalase, $\mathbf{g}$ SOD1, h SOD3, using quantitative PCR; beta actin served as loading control in western blots, representative blots are shown. At least three independent experiments in biological triplicates were performed, scr scrambled control

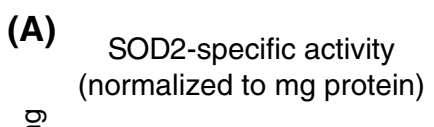

(B) SOD2 activity

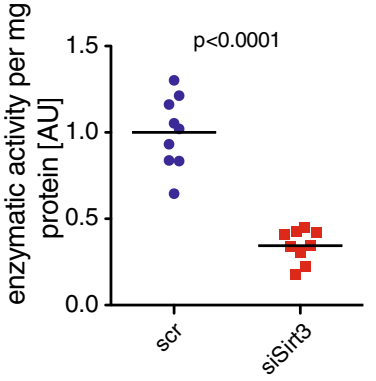

(C)

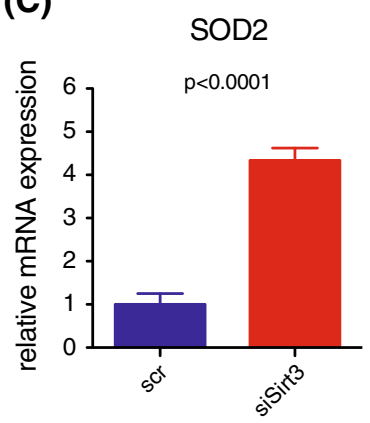

(D)

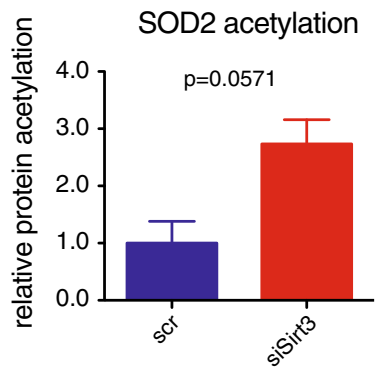

(F)
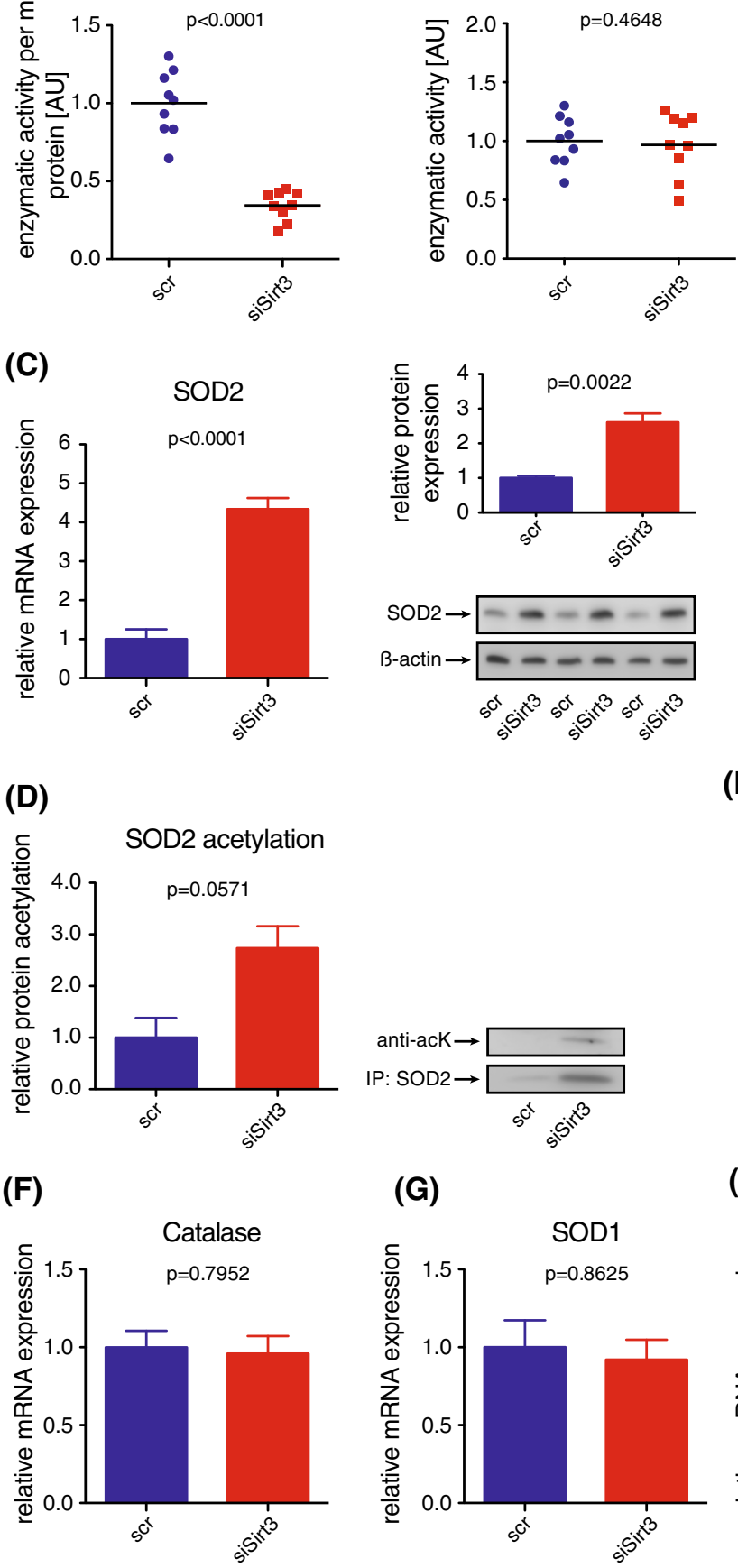

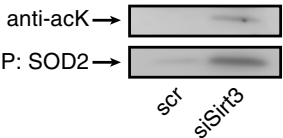

(G)

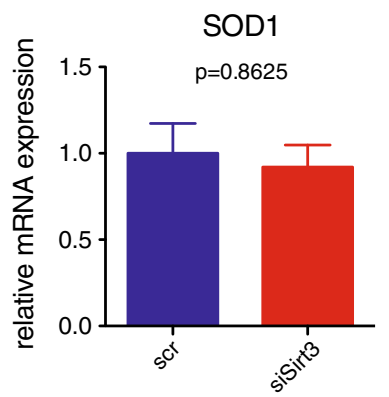

(E) SOD2 nitrosylation

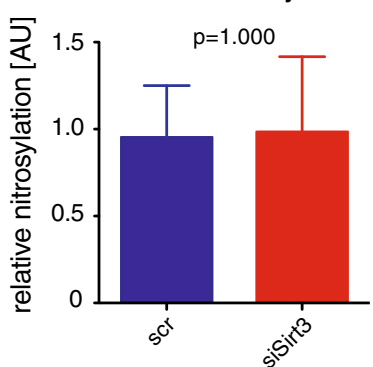

(H)

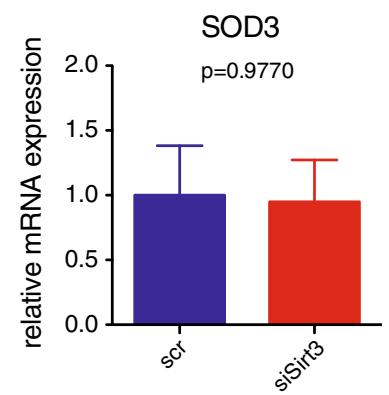

\section{Transcriptional induction of SOD2 upon transient knockdown of Sirt3 is C/EBP-p-dependent}

To shed light on the unexpected transcriptional upregulation of endothelial SOD2 during transient knockdown of Sirt3, we assessed the expression levels of known, acetylation-dependent transcription factors of SOD2. Expression levels of Sp1 and STAT3 were unaffected by transient knockdown of Sirt3 (Fig. 4a, b), whereas expression of CCAAT/enhancer binding protein beta $(\mathrm{C} / \mathrm{EBP}-\beta)$ was increased (Fig. 4c).

This differential expression in response to decreased Sirt3 levels prompted us to address the relevance of C/EBP- $\beta$ in the transcriptional induction of SOD2 during 
(A)

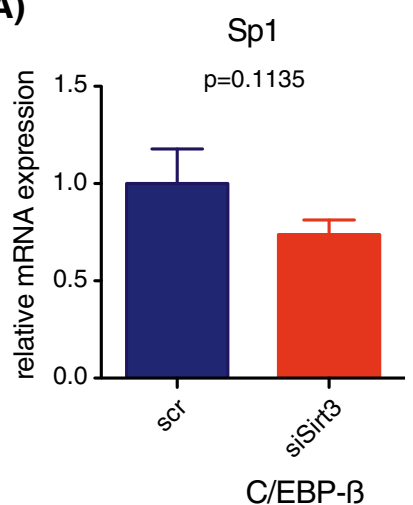

(D)

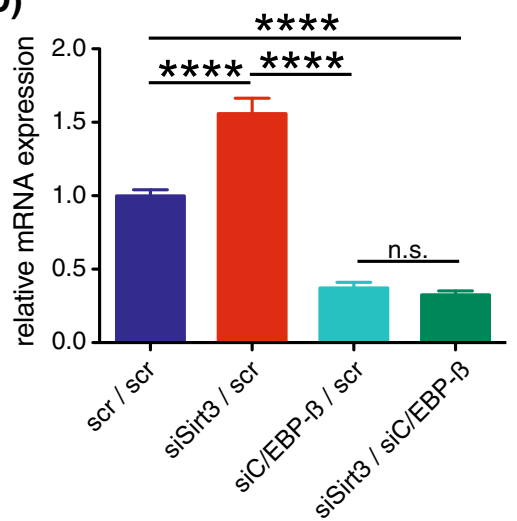

(F)

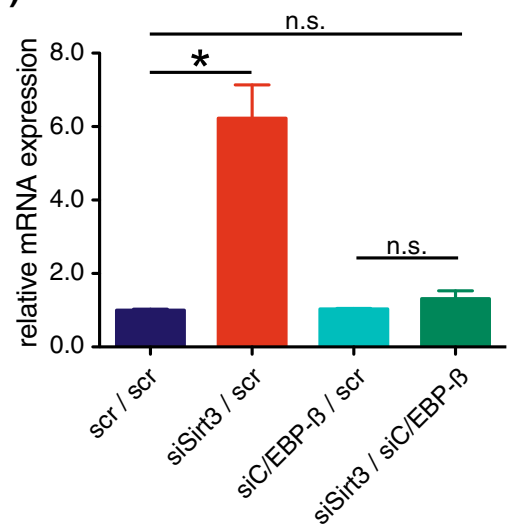

Fig. 4 Transcriptional induction of SOD2 upon transient knockdown of Sirt3 is C/EBP- $\beta$-dependent. a-d Expression analyses of the transcription factors a Sp1, b STAT3, c, d C/EBP- $\beta$ using quantitative PCR in HAEC following siRNA-mediated knockdown of Sirt3, and d single or simultaneous transient knockdown of C/EBP- $\beta$ and Sirt3, respectively. e Western blot analysis of C/EBP- $\beta$ in HAEC following single and simultaneous transient knockdown of C/EBP- $\beta$ and Sirt3, respectively. f, $\mathbf{g}$ Expression analyses of SOD2 in HAEC following

transient knockdown of Sirt3. Interestingly, simultaneous knockdown of Sirt3 and C/EBP- $\beta$ abrogated the transcriptional upregulation of SOD2, whereas single knockdown of C/EBP- $\beta$ had no effect on SOD2 expression levels compared with controls (Fig. 4d-g).
(C)

\section{C/EBP-B}

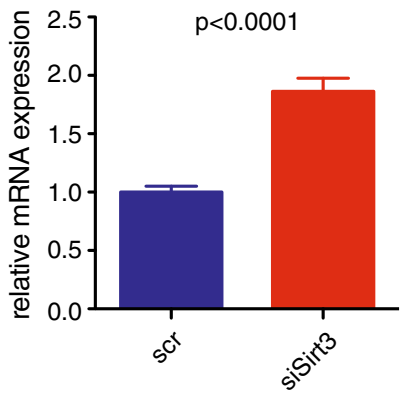

C/EBP-B

(E)
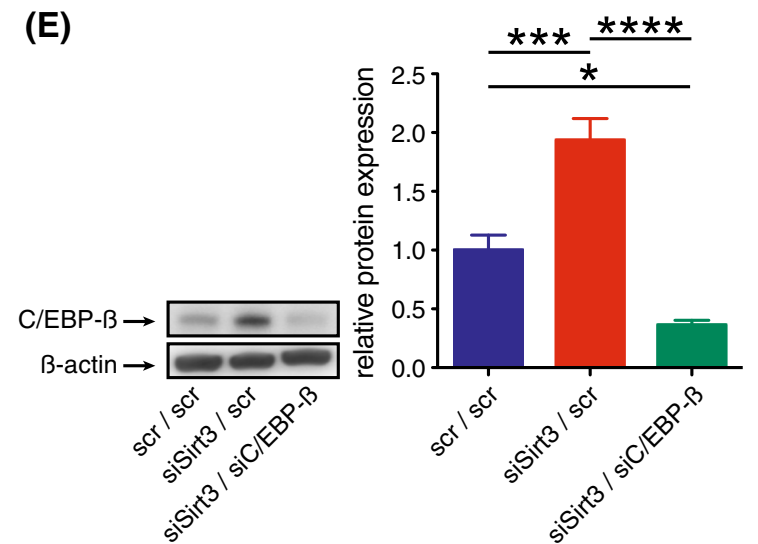

(G)

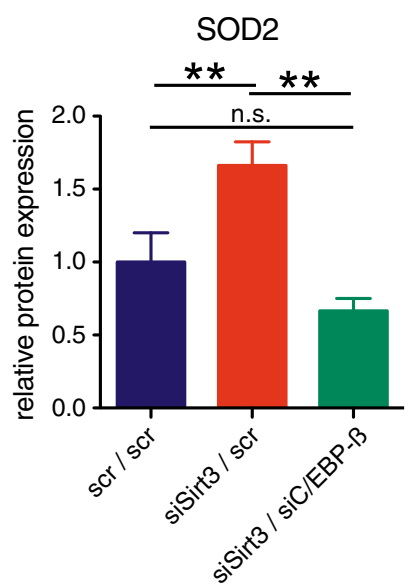

single or simultaneous transient knockdown of C/EBP- $\beta$ and Sirt3, respectively, using $\mathbf{f}$ quantitative $\mathrm{PCR}$ and $\mathbf{g}$ western blot analysis. At least three independent experiments in biological triplicates were performed, scr scrambled control, $\mathbf{d}-\mathbf{g} p$ values indicate overall significance by Kruskal-Wallis (d-f) or one-way ANOVA tests (g), differences between single groups by Dunn's (d-f) or Bonferroni (g) post hoc tests are indicated separately: n.s. non-significant, $* p<0.05, * * p<0.01$

\section{Transcriptional induction of C/EBP- $\beta$ is SOD2- dependent}

To assess the role of SOD2 on the transcriptional induction of C/EBP- $\beta$ we used a loss-of-function approach in HAEC. 
Transient knockdown of SOD2 was associated with a significant increase in C/EBP- $\beta$ transcription on RNA-level (Fig S5A), which translated into a trend towards increased protein levels of C/EBP- $\beta$ (Fig S5B), indicating the existence of a direct feedback loop between SOD2 and its transcription factor $\mathrm{C} / \mathrm{EBP}-\beta$ in endothelial cells. Sirt3 expression was unaltered (Fig S5 F, G).

\section{Scavenging mitochondrial superoxide does not affect Sirt3-dependent transcriptional induction of SOD2}

To investigate whether SOD2 induction upon Sirt3 deficiency is superoxide-dependent, mitochondrial superoxide was scavenged using the mitochondrial-targeted superoxide scavenger mitoTEMPO. Mitochondrial superoxide accumulation following knockdown of Sirt3 in HAEC was successfully blunted by mitoTEMPO, as assessed by fluorescence imaging after MitoSOX staining (Fig. 5a-c). Interestingly, SOD2 induction upon Sirt3 knockdown was unaffected by blunting mitochondrial superoxide accumulation (Fig. 5d). Transcriptional upregulation of C/EBP- $\beta$ following knockdown of Sirt3 was also unaltered upon scavenging of mitochondrial superoxide (Fig. 5e). Translation to increased protein levels could not be observed, independent of mitochondrial superoxide (Fig. 5f).

\section{Interruption of the physiological C/EBP- $\beta$ - dependent feedback regulation of endothelial SOD2 exacerbates mitochondrial superoxide levels and culminates in endothelial cell death}

To reveal the functional relevance of the C/EBP- $\beta$-dependent transcriptional feedback regulation of endothelial SOD2 upon Sirt3 deficiency, we assessed mitochondrial superoxide levels following single or simultaneous knockdown of C/EBP- $\beta$ and Sirt3, respectively, compared with sham-transfected controls. Concomitant with the abrogation of the transcriptional induction of SOD2 following simultaneous knockdown of C/EBP- $\beta$ and Sirt3, mitochondrial superoxide levels were further enhanced compared with single-knockdown controls (Fig. 6a, b). Transient knockdown of C/EBP- $\beta$ alone had no effect on mitochondrial superoxide levels (Fig. 6a, b). Interestingly, we observed an increased cell death upon prolonged cultivation $(40 \mathrm{~h})$ following simultaneous knockdown of C/EBP- $\beta$ and Sirt3 that occurred in none of the control conditions (Fig. 6c, d): Incubation for up to $40 \mathrm{~h}$ following knockdown led to a demise of the majority of cells (Fig. 6d), which we interpret as the consequence of increased oxidative stress.

\section{Discussion}

\section{Principle findings}

We identify Sirt3 as an important player in the homeostasis of endothelial mitochondrial superoxide. Under physiological conditions, endogenous endothelial Sirt3 appears to uphold SOD2 activity by maintaining its deacetylation for effective degradation of mitochondrial superoxide and thereby preserving normal endothelial function. Lack of Sirt3 is associated with a hyperacetylation of SOD2. A concomitant loss of SOD2 activity is, however, compensated for by a C/EBP- $\beta$ dependent induction of SOD2 expression. The latter occurs independent of mitochondrial superoxide accumulation, yet potentially upon an initial transcriptional repression of SOD2 upon Sirt3 deficiency. Consequently, endothelial function is maintained under normal circumstances. Only upon a high-cholesterol diet, leading to increased oxidative stress, a mild, superoxidedependent impairment of endothelial function can be unmasked. In vitro experiments in human aortic endothelial cells indicate that this may be due to an accumulation of mitochondrial superoxide secondary to a residual moderate imbalance of mitochondrial superoxide generation and detoxification. Since none of the superoxide scavenging or generating enzymes assessed were altered upon Sirt3 deficiency, we extrapolate that impaired mitochondrial function upon Sirt3 deficiency [38, 54] may increase mitochondrial superoxide formation.

\section{Added value}

Anti-oxidative effects of Sirt3 have been described in a variety of contexts including age-related hearing loss [49], embryogenesis [22], neuronal injury [10], exercise training [27], and cardiac hypertrophy [51]. In the majority of these settings, the protective effects of Sirt 3 are mediated by an augmented radical scavenging through SOD2 and/or catalase. It remains controversial whether these effects are brought about by a transcriptional induction of either of these two ROS detoxification systems or by their Sirt3dependent deacetylation and consecutive activation [8, 42, 51].

In the present study, we report on the role of endogenous Sirt3 in human aortic endothelial cells and its functional effects on endothelium-dependent vasodilation in a mouse model applying a genetic loss-of-function approach. Corroborating previous reports on anti-oxidative effects of Sirt3 [42], transient knockdown of endothelial Sirt3 abrogated the superoxide scavenging capacity of SOD2 and increased its acetylation. 
(A)

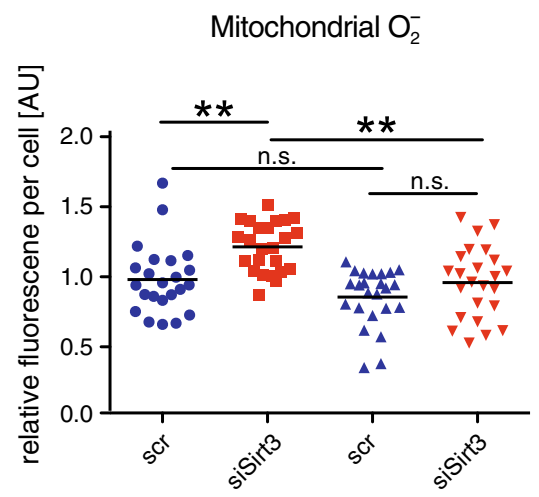

mitoTEMPO

vehicle

(C)

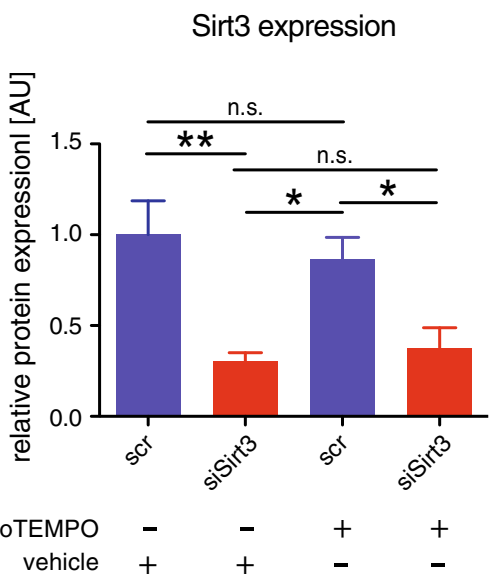

(E)

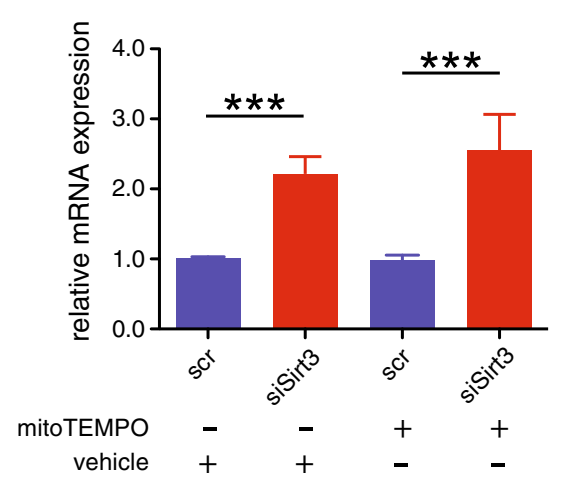

Fig. 5 Scavenging mitochondrial superoxide does not affect Sirt3dependent transcriptional induction of SOD2. a Quantification of mitochondrial superoxide per cell, as visualized by MitoSOX ${ }^{\mathrm{TM}}$ staining, using fluorescence imaging of HAEC following transient knockdown of Sirt3 or transfection with scrambled siRNA (scr) in presence or absence of the mitochondrial-targeted anti-oxidant mitoTEMPO. b Representative micrographs of the setup described

In contrast to previous data [51], we observed that transcription of endothelial SOD2 was not reduced but enhanced following transient knockdown of Sirt3,

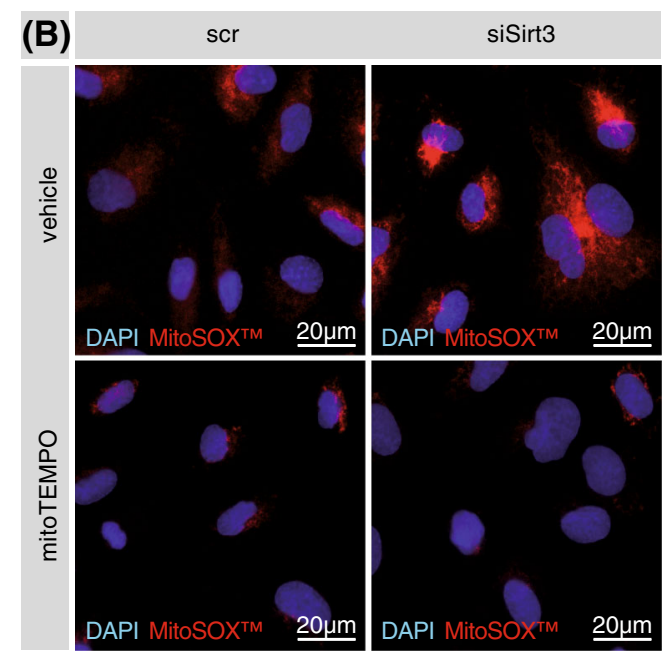

(D)

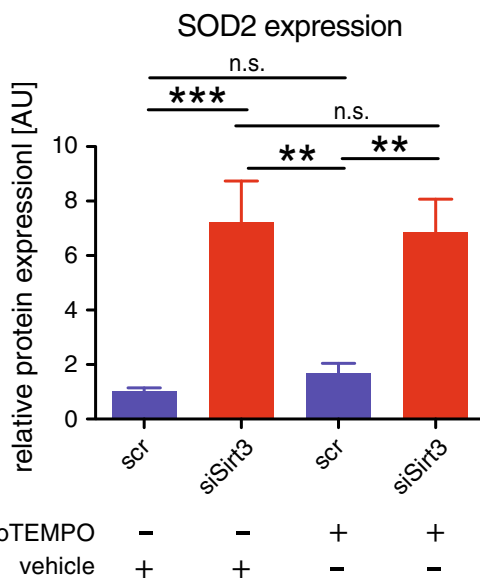

(F)

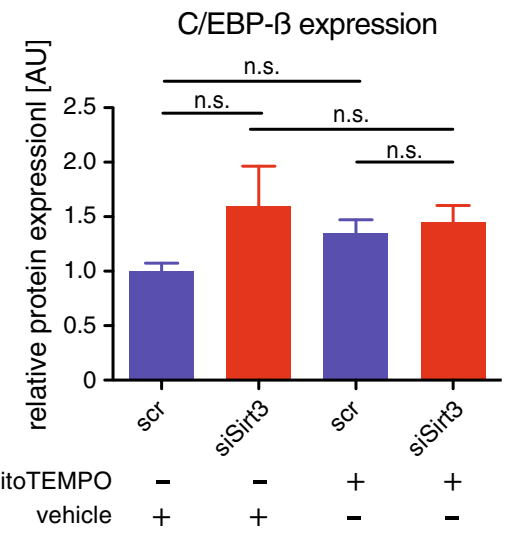

in a, showing nuclei (blue, DAPI) and mitochondrial superoxide ( $\mathrm{red}$, MitoSOX $\left.{ }^{\mathrm{TM}}\right)$. $\mathbf{c}, \mathbf{d}, \mathbf{f}$ Quantification of western blot analyses of Sirt3 (c), SOD2 (d), and C/EBP- $\beta$ (f). e quantitative PCR of C/EBP- $\beta$. At least three independent experiments in biological triplicates were performed. Scale bars $20 \mu \mathrm{m}$, DAPI 4'-6-diamidin-2-phenylindol, n.s. non-significant, $* * p<0.01, * * * p<0.001$

compensating for its decreased enzymatic activity. However, an initial transcriptional repression of SOD2 upon Sirt3 deficiency, preceding the C/EBP- $\beta$ dependent 
transcriptional induction of SOD2 cannot be excluded. Even though the drop in endothelial SOD2 activity upon Sirt3 deficiency slightly exceeded its transcriptional increase, we extrapolate that an excess in mitochondrial superoxide generation secondary to a Sirt3-dependent impairment of mitochondrial function [38] may contribute to the disequilibrium between mitochondrial superoxide generation and detoxification. Of note, expression levels of other endothelial ROS and reactive nitrogen species detoxification systems, including catalase and the thioredoxin system, were unaffected by transient knockdown of Sirt3. Therefore, a thioredoxin-mediated transcriptional induction of SOD2, as reported in yeast and primary human lung microvascular endothelial cells [11, 39] appears unlikely. Assessment of endothelial nitric oxide synthase (eNOS) uncovered a decreased phosphorylation of Thr495 as well as an increased coupling following transient knockdown of Sirt3, equivalent to an increased enzymatic activity [43, 52]. Importantly, NO generation remained unchanged, indicating that increased activity remains functionally irrelevant with regard to NO homeostasis. However, increased eNOS coupling may contribute to counteract increased ROS levels upon Sirt3 deficiency.

Moreover, our in vitro experiments identify mitochondria as the compartment exhibiting increased ROS in the absence of endothelial Sirt3, a finding, for which to date only indirect evidence exists [22, 54].

Elegant promoter studies identified that C/EBP- $\beta$ is necessary to align with an intronic enhancer of SOD2 to facilitate its transcription in response to increased levels of intracellular ROS $[6,21,30]$. The differential regulation of C/EBP- $\beta$ in response to transient Sirt3 knockdown and its acetylation-dependent binding capacity [6] prompted us to investigate the role of C/EBP- $\beta$ in the transcriptional regulation of SOD2 in the absence of endothelial Sirt3. Abrogation of SOD2 induction upon simultaneous knockdown of both Sirt3 and C/EBP- $\beta$ exacerbated mitochondrial superoxide accumulation and culminated in endothelial cell death after prolonged cultivation. Interestingly, we observed a bidirectional feedback regulation between C/EBP- $\beta$ and SOD2 with an SOD2-dependent transcriptional induction of C/EBP- $\beta$ and vice versa. This might indicate that an initial transcriptional repression of SOD2 upon Sirt3-deficiency, as has been reported by others $[20,24,45,51]$, in addition to a blunted SOD2 activity, may have preceded C/EBP- $\beta$ dependent transcriptional induction of SOD2. Importantly, transcriptional induction of C/EBP- $\beta$ was independent of mitochondrial superoxide. These findings highlight the functional relevance of this novel C/EBP- $\beta$-dependent transcriptional induction of SOD2 in absence of Sirt3 in human aortic endothelial cells.
The ex vivo assessment of endothelium-dependent vessel relaxation showed a rather atypically shaped relaxation curve; the initial sigmoidal shape is interrupted by a weak contraction, starting at acetylcholine levels around $1 \mu \mathrm{M}$, before reaching complete relaxation at $100 \mu \mathrm{M}$. Interestingly, this intermittent contraction was exacerbated in mice fed a high-cholesterol diet. This and the fact that this phenomenon disappeared in presence of PEG-SOD indicate that this intermittent contraction in response to higher acetylcholine dosages is an oxidative stress-dependent phenomenon, as we have observed previously [60].

\section{Potential limitations}

This study has to be interpreted in light of the following limitations: The difference in endothelium-dependent vasorelaxation between the two genotypes is mild. However, rescue of this phenotype by scavenging of superoxide via exogenous pegylated superoxide dismutase proves superoxide-dependency. Furthermore, improvement of the sensitivity to ACh of both genotypes upon enhanced superoxide scavenging highlights the tight regulation of endothelial function by superoxide and $\mathrm{NO}$ and stresses the physiological relevance of Sirt3 and C/EBP- $\beta$ in regulation of endothelial SOD2 activity and thus of endothelial function.

In light of the $C / E B P-\beta$ dependent transcriptional compensation of the Sirt3-dependent loss of SOD2 activity in concert with unchanged expression levels of other superoxide generators or scavengers, our data do not provide an explanation for the increased mitochondrial superoxide levels upon Sirt3 deficiency. The extrapolation that a known Sirt3-dependent impairment of the mitochondrial function may enhance mitochondrial superoxide generation remains speculative and warrants further investigation.

In addition, we would like to point out that endothelial dysfunction in Sirt $3^{-/-}$mice was observed only upon exposure to a high-cholesterol diet, known to induce oxidative stress [37]. In vitro, mitochondrial superoxide accumulation and differential SOD2 regulation upon Sirt3 deficiency was apparent under basal conditions. Though we assume that this is due to the nature of in vitro setups in general, extrapolation to our ex vivo data may be limited.

Moreover, endothelial-dependent vasodilation was assessed using mouse aortic rings in organ chamber baths, which is an ex vivo approach. Again, extrapolation to in vivo vascular function as well as to other species has to be done with caution. 


\section{(A)}

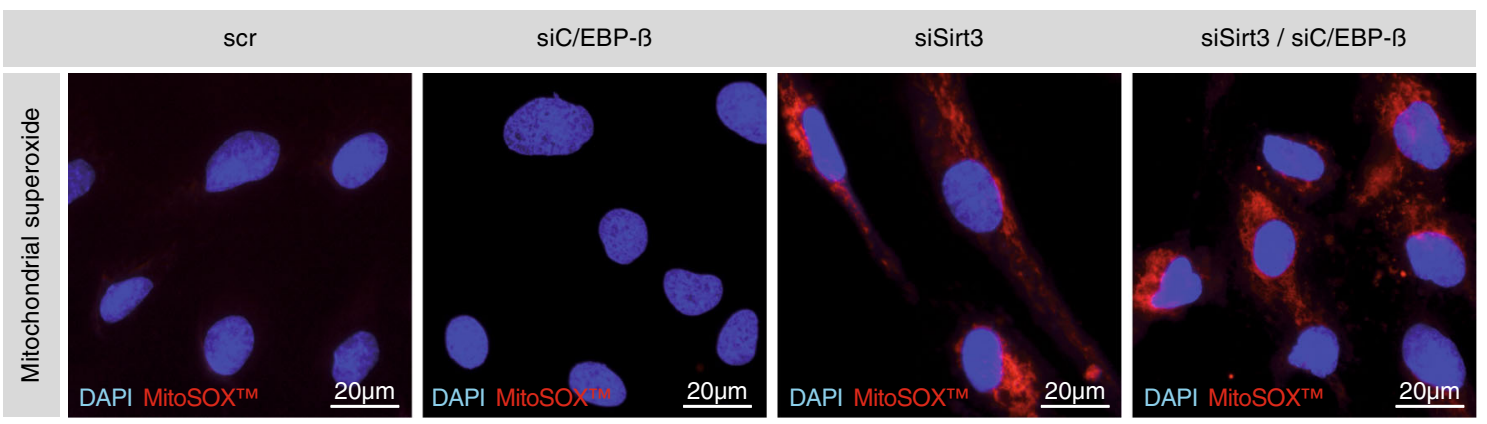

(B)
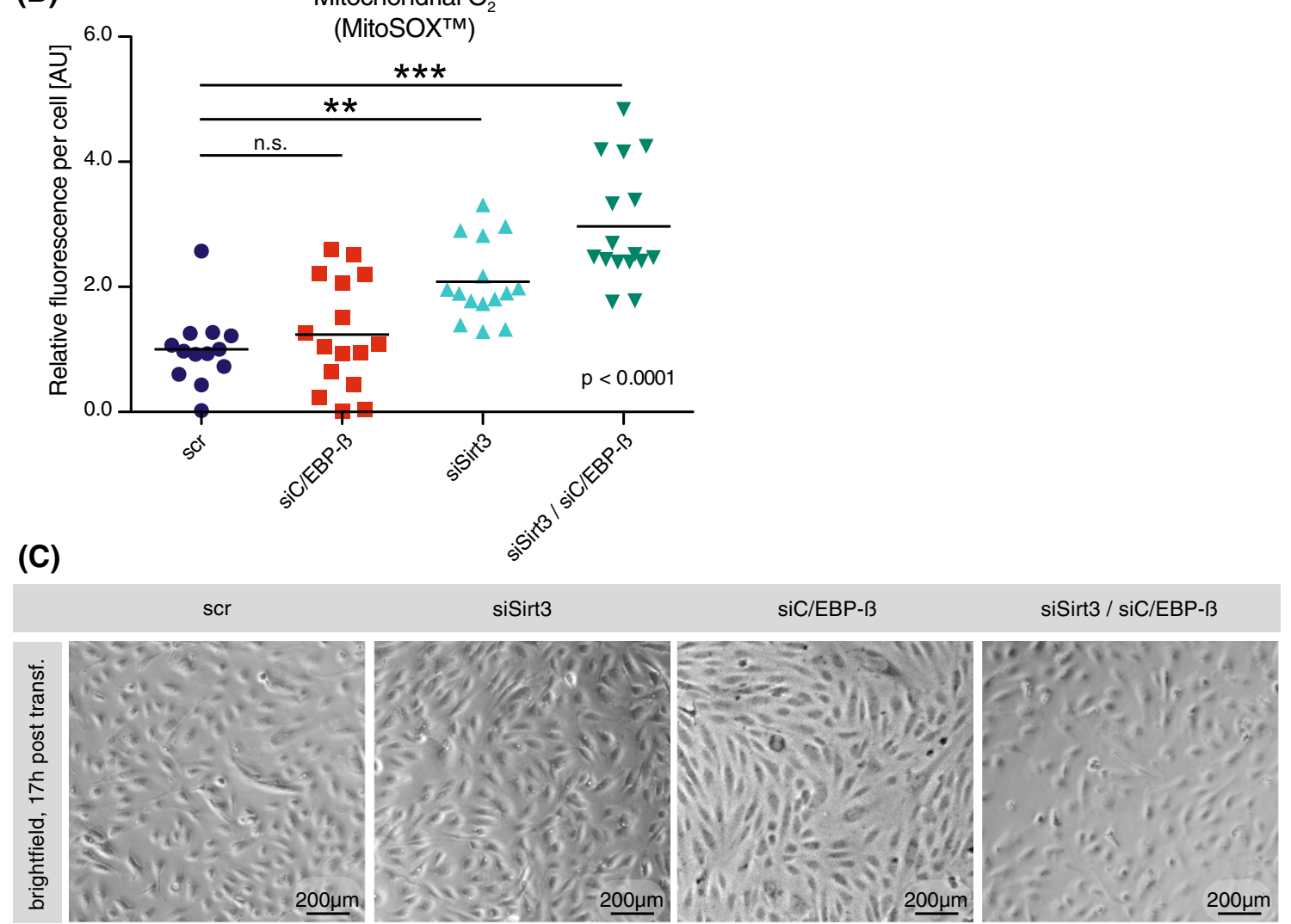

(D)

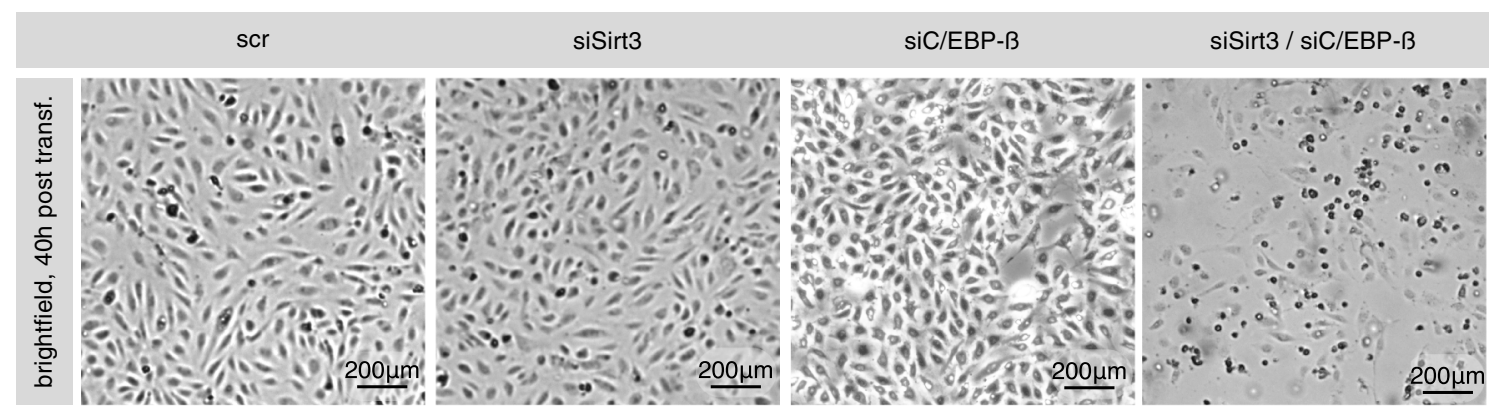


4Fig. 6 Interruption of the physiological C/EBP- $\beta$-dependent transcriptional feedback regulation of SOD2 during transient knockdown of Sirt3 exacerbates mitochondrial superoxide formation and culminates in endothelial cell death. a Fluorescence imaging of HAEC following single and simultaneous transient knockdown of C/EBP- $\beta$ and Sirt3, respectively. Representative micrographs show nuclei (blue) and mitochondrial superoxide (red, MitoSOX ${ }^{\mathrm{TM}}$ ), the latter visualized by the mitochondrial- and superoxide-specific fluorescent MitoSOX ${ }^{\mathrm{TM}}$ probe. Scale bars $20 \mu \mathrm{m}$. b Quantification of mitochondrial superoxide per cell; medians and single data points are shown. c, d Representative brightfield phase-contrast micrographs of cultured HAEC $17 \mathrm{~h} \mathrm{(c)} \mathrm{and} 40 \mathrm{~h}$ (d) after transient knockdown of Sirt3 and C/EBP- $\beta$, either alone or in combination; scale bars $200 \mu \mathrm{m}$. At least three independent experiments in biological triplicates were performed, scr scrambled control, DAPI 4'-6-diamidin-2-phenylindol, $n . s$. non-significant, $* p<0.05, * * p<0.01, * * * p<0.001$

\section{Conclusions and implications}

The current data indicate a protective role of endogenous endothelial Sirt3 in mice fed a high-cholesterol diet, maintaining endothelium-dependent vasorelaxation. The in vitro findings suggest that a novel C/EBP- $\beta$-dependent rescue mechanism diminishes Sirt3-dependent endothelial dysfunction under physiological conditions (normal diet).

We have reported Sirt3-mediated protection from accelerated weight gain and a decline in metabolic flexibility [55], two important risk factors of human cardiovascular diseases [5]. Our current findings identify an interplay of Sirt3, SOD2, and C/EBP- $\beta$ in the endothelial redox system. Endothelial dysfunction is independently associated with future adverse cardiovascular events $[16,17,36,40,48]$. Further research is warranted to better understand the putatively protective role of Sirt3 in human cardiovascular disease.

Acknowledgments This work was funded by the Swiss National Science Foundation to JA (31003A-140780), to TFL (310030-135815), and to CMM (146923, 140336), the National Institute of Health (US) to JA (R01AG043930), Systems X (SysX.ch) to JA (2013-15), the University Research Priority Program Integrative Human Physiology at the University of Zurich to TFL and CMM, Matching Funds by the University of Zurich to SW and CMM, the Ecole Polytechnique Fédérale de Lausanne to JA, and the Zurich Heart House-Foundation for Cardiovascular Research, Zurich, Switzerland.

\section{Compliance with ethical standards}

Conflict of interest None.

Open Access This article is distributed under the terms of the Creative Commons Attribution 4.0 International License (http://creative commons.org/licenses/by/4.0/), which permits unrestricted use, distribution, and reproduction in any medium, provided you give appropriate credit to the original author(s) and the source, provide a link to the Creative Commons license, and indicate if changes were made.

\section{References}

1. Ahn B-H, Kim H-S, Song S, Lee IH, Liu J, Vassilopoulos A, Deng C-X, Finkel T (2008) A role for the mitochondrial deacetylase Sirt3 in regulating energy homeostasis. Proc Natl Acad Sci USA 105:14447-14452. doi:10.1073/pnas. 0803790105

2. Alwan A, Armstron T, Bettcher D, Branca F, Chisholm D, Garfileld R, MacLean D, Mathers C, Mendis S, Poznyak V, Riley L, Tang CT, Wild C (2011) Global status report on noncommunicable diseases. WHO Press, World Health Organization, Geneva. doi:10.2471/BLT.11.091074

3. Bell EL, Guarente L (2011) The SirT3 divining rod points to oxidative stress. Mol Cell 42:561-568. doi:10.1016/j.molcel. 2011.05.008

4. Bellizzi D, Rose G, Cavalcante P, Covello G, Dato S, De Rango F, Greco V, Maggiolini M, Feraco E, Mari V, Franceschi C, Passarino G, De Benedictis G (2005) A novel VNTR enhancer within the SIRT3 gene, a human homologue of SIR2, is associated with survival at oldest ages. Genomics 85:258-263. doi:10. 1016/j.ygeno.2004.11.003

5. Berry JD, Dyer A, Cai X, Garside DB, Ning H, Thomas A, Greenland P, Van Horn L, Tracy RP, Lloyd-Jones DM (2012) Lifetime risks of cardiovascular disease. $\mathrm{N}$ Engl $\mathrm{J}$ Med 366:321-329. doi:10.1056/NEJMoa1012848

6. Cesena TI, Cardinaux JR, Kwok R, Schwartz J (2007) CCAAT/ enhancer-binding protein $(\mathrm{C} / \mathrm{EBP})$ beta is acetylated at multiple lysines: acetylation of $\mathrm{C} / \mathrm{EBP}$ beta at lysine 39 modulates its ability to activate transcription. J Biol Chem 282:956-967. doi:10.1074/jbc.M511451200

7. Chalkiadaki A, Guarente L (2012) Sirtuins mediate mammalian metabolic responses to nutrient availability. Nat Rev Endocrinol 8:287-296. doi:10.1038/nrendo.2011.225

8. Chen Y, Zhang J, Lin Y, Lei Q, Guan K-L, Zhao S, Xiong Y (2011) Tumour suppressor SIRT3 deacetylates and activates manganese superoxide dismutase to scavenge ROS. EMBO Rep 12:534-541. doi:10.1038/embor.2011.65

9. Claycombe KJ, Roemmich JN, Johnson L, Vomhof-DeKrey EE, Johnson WT (2015) Skeletal muscle Sirt3 expression and mitochondrial respiration are regulated by a prenatal low-protein diet. J Nutr Biochem 26:184-189. doi:10.1016/j.jnutbio.2014.10.003

10. Dai S-H, Chen T, Wang Y-H, Zhu J, Luo P, Rao W, Yang Y-F, Fei Z, Jiang X-F (2014) Sirt3 protects cortical neurons against oxidative stress via regulating mitochondrial $\mathrm{Ca}^{2+}$ and mitochondrial biogenesis. Int J Mol Sci 15:14591-14609. doi:10. 3390/ijms 150814591

11. Das KC, Lewis-Molock Y, White CW (1997) Elevation of manganese superoxide dismutase gene expression by thioredoxin. Am J Respir Cell Mol Biol 17:713-726. doi:10.1165/ajrcmb.17.6. 2809

12. Fernandez-Marcos PJ, Jeninga EH, Canto C, Harach T, de Boer VCJ, Andreux P, Moullan N, Pirinen E, Yamamoto H, Houten SM, Schoonjans K, Auwerx J (2012) Muscle or liver-specific Sirt3 deficiency induces hyperacetylation of mitochondrial proteins without affecting global metabolic homeostasis. Sci Rep 2:425. doi:10.1038/srep00425

13. Giblin W, Skinner ME, Lombard DB (2014) Sirtuins: guardians of mammalian healthspan. Trends Genet 30:271-286. doi:10. 1016/j.tig.2014.04.007

14. Haigis MC, Sinclair DA (2010) Mammalian sirtuins: biological insights and disease relevance. Annu Rev Pathol Mech 5:253-295. doi:10.1146/annurev.pathol.4.110807.092250

15. Halaschek-Wiener J, Amirabbasi-Beik M, Monfared N, Pieczyk M, Sailer C, Kollar A, Thomas R, Agalaridis G, Yamada S, Oliveira L (2009) Genetic variation in healthy oldest-old. PLoS One 4:e6641. doi:10.1371/journal.pone.0006641.t004

16. Halcox JPJ, Schenke WH, Zalos G, Mincemoyer R, Prasad A, Waclawiw MA, Nour KRA, Quyyumi AA (2002) Prognostic value of coronary vascular endothelial dysfunction. Circulation 106:653-658. doi:10.1161/01.CIR.0000025404.78001.D8 
17. Heitzer T, Schlinzig T, Krohn K, Meinertz T, Munzel T (2001) Endothelial dysfunction, oxidative stress, and risk of cardiovascular events in patients with coronary artery disease. Circulation 104:2673-2678. doi:10.1161/hc4601.099485

18. Hirschey MD, Shimazu T, Jing E, Grueter CA, Collins AM, Aouizerat B, Stančáková A, Goetzman E, Lam MM, Schwer B, Stevens RD, Muehlbauer MJ, Kakar S, Bass NM, Kuusisto J, Laakso M, Alt FW, Newgard CB, Farese RV, Kahn CR, Verdin E (2011) SIRT3 deficiency and mitochondrial protein hyperacetylation accelerate the development of the metabolic syndrome. Mol Cell 44:177-190. doi:10.1016/j.molcel.2011.07.019

19. Houtkooper RH, Pirinen E, Auwerx J (2012) Sirtuins as regulators of metabolism and healthspan. Nat Rev Mol Cell Biol 13:225-238. doi:10.1038/nrm3293

20. Jacobs KM, Pennington D, Bisht KS, Aykin-Burns N, Kim H-S, Mishra M, Sun L, Nguyen P, Ahn B-H, Leclerc J, Deng C-X, Spitz DR, Gius D (2008) SIRT3 interacts with the daf-16 homolog FOXO3a in the mitochondria, as well as increases FOXO3a dependent gene expression. Int J Biol Sci 4:291-299

21. Jones PL, Ping D, Boss JM (1997) Tumor necrosis factor alpha and interleukin-1beta regulate the murine manganese superoxide dismutase gene through a complex intronic enhancer involving C/EBP-beta and NF-kappaB. Mol Cell Biol 17:6970-6981

22. Kawamura Y, Uchijima Y, Horike N, Tonami K, Nishiyama K, Amano T, Asano T, Kurihara Y, Kurihara H (2010) Sirt3 protects in vitro-fertilized mouse preimplantation embryos against oxidative stress-induced p53-mediated developmental arrest. J Clin Invest 120:2817-2828. doi:10.1172/JCI42020DS1

23. Koentges C, Pfeil K, Schnick T, Wiese S, Dahlbock R, Cimolai MC, Meyer-Steenbuck M, Cenkerova K, Hoffmann MM, Jaeger C, Odening KE, Kammerer B, Hein L, Bode C, Bugger H (2015) SIRT3 deficiency impairs mitochondrial and contractile function in the heart. Basic Res Cardiol 110:36. doi:10.1007/s00395-015-0493-6

24. Kwon DN, Park WJ, Choi YJ, Gurunathan S, Kim JH (2015) Oxidative stress and ROS metabolism via down-regulation of sirtuin 3 expression in Cmah-null mice affect hearing loss. Aging (Albany) 7:579-594

25. Lane MA, Mattison J, Ingram DK, Roth GS (2002) Caloric restriction and aging in primates: relevance to humans and possible CR mimetics. Microsc Res Tech 59:335-338. doi:10.1002/ jemt. 10214

26. Laslett LJ, Alagona P Jr, Clark BA III, Drozda JP Jr, Saldivar F, Wilson SR, Poe C, Hart M (2012) The worldwide environment of cardiovascular disease: prevalence, diagnosis, therapy, and policy issues. J Am Coll Cardiol 60:S1-S49. doi:10.1016/j.jacc.2012.11. 002

27. Lin L, Chen K, Khalek WA, Ward JL, Yang H, Chabi B, Wrutniak-Cabello C, Tong Q (2014) Regulation of skeletal muscle oxidative capacity and muscle mass by SIRT3. PLoS One 9:e85636. doi:10.1371/journal.pone.0085636.s005

28. Madamanchi NR, Runge MS (2007) Mitochondrial dysfunction in atherosclerosis. Circ Res 100:460-473. doi:10.1161/01.RES. 0000258450.44413 .96

29. Madamanchi NR, Vendrov A, Runge MS (2005) Oxidative stress and vascular disease. Arterioscler Thromb Vasc Biol 25:29-38. doi:10.1161/01.RES.0000258450.44413.96

30. Maehara K, Hasegawa T, Xiao H, Takeuchi A, Abe R, Isobe K-I (1999) Cooperative interaction of NF- $\mathrm{KB}$ and C/EBP binding sites is necessary for manganese superoxide dismutase gene transcription mediated by lipopolysaccharide and interferon- $\gamma$. FEBS Lett 449:115-119. doi:10.1016/S0014-5793(99)00408-1

31. Mailloux RJ, Bériault R, Lemire J, Singh R, Chénier DR, Hamel RD, Appanna VD (2007) The tricarboxylic acid cycle, an ancient metabolic network with a novel twist. PLoS One 2:e690. doi:10. 1371/journal.pone.0000690.g005
32. McCay CM, Crowell MF, Maynard LA (1935) The effect of retarded growth upon the length of life span and upon the ultimate body size. J Nutr 10:63-79

33. Mercer JR, Cheng KK, Figg N, Gorenne I, Mahmoudi M, Griffin J, Vidal-Puig A, Logan A, Murphy MP, Bennett M (2010) DNA damage links mitochondiral dysfunction to atherosclerosis and the metabolic syndrome. Circ Res 107:1021-1031. doi:10.1161/ CIRCRESAHA.110.218966

34. Mouchiroud L, Houtkooper RH, Moullan N, Katsyuba E, Ryu D, Canto C, Mottis A, Jo Y-S, Viswanathan M, Schoonjans K, Guarente L, Auwerx J (2013) The $\mathrm{NAD}^{+} /$sirtuin pathway modulates longevity through activation of mitochondrial UPR and FOXO signaling. Cell 154:430-441. doi:10.1016/j.cell.2013.06.016

35. Mortuza R, Chen S, Feng B, Sen S, Chakrabarti S (2013) High glucose induced alteration of SIRTs in endothelial cells causes rapid aging in a P300 and FOXO regulated pathway. PLoS One 8:e54514. doi:10.1371/journal.pone.0054514.s003

36. Neunteufl T, Heher S, Katzenschlager R, Wölfl G, Kostner K, Maurer G, Weidinger F (2000) Late prognostic value of flowmediated dilation in the brachial artery of patients with chest pain. Am J Cardiol 86:207-210. doi:10.1016/S00029149(00)00857-2

37. Osto E, Matter CM, Kouroedov A, Malinski T, Bachschmid M, Camici GG, Kilic U, Stallmach T, Boren J, Iliceto S, Luscher TF, Cosentino F (2008) c-Jun N-terminal kinase 2 deficiency protects against hypercholesterolemia-induced endothelial dysfunction and oxidative stress. Circulation 118:2073-2080. doi:10.1161/ CIRCULATIONAHA.108.765032

38. Paulin R, Dromparis P, Sutendra G, Gurtu V, Zervopoulos S, Bowers L, Haromy A, Webster L, Provencher S, Bonnet S, Michelakis ED (2014) Sirtuin 3 deficiency is associated with inhibited mitochondrial function and pulmonary arterial hypertension in rodents and humans. Cell Metab 20:837-839. doi:10. 1016/j.cmet.2014.08.011

39. Pedrajas JR, Carreras A, Valderrama R, Barroso JB (2010) Mitochondrial 1-Cys-peroxiredoxin/thioredoxin system protects manganese-containing superoxide dismutase (Mn-SOD) against inactivation by peroxynitrite in Saccharomyces cerevisiae. NATO Sci S A Life Sci 23:206-213. doi:10.1016/j.niox.2010.06.004

40. Perticone F, Ceravolo R, Pujia A, Ventura G, Iacopino S, Scozzafava A, Ferraro A, Chello M, Mastroroberto P, Verdecchia P, Schillaci G (2001) Prognostic significance of endothelial dysfunction in hypertensive patients. Circulation 104:191-196. doi:10.1161/01.CIR.104.2.191

41. Puddu P, Puddu GM, Cravero E, De Pascalis S, Muscari A (2009) Emerging role of cardiovascular risk factor-induced mitochondrial dysfunction in atherogenesis. J Biomed Sci 16:112. doi:10. 1186/1423-0127-16-112

42. Qiu X, Brown K, Hirschey MD, Verdin E, Chen D (2010) Calorie restriction reduces oxidative stress by SIRT3-mediated SOD2 activation. Cell Metab 12:662-667. doi:10.1016/j.cmet.2010.11. 015

43. Rabelink TJ, Lüscher TF (2006) Endothelial nitric oxide synthase: host defense enzyme of the endothelium? Arterioscler Thromb Vas 26:267-271. doi:10.1161/01.ATV.0000196554. 85799.77

44. Rahman M, Nirala NK, Singh A, Zhu LJ, Taguchi K, Bamba T, Fukusaki E, Shaw LM, Lambright DG, Acharya JK, Acharya UR (2014) Drosophila Sirt2/mammalian SIRT3 deacetylates ATP synthase and regulates complex $\mathrm{V}$ activity. J Cell Biol 206:289-305. doi:10.1186/1471-2105-11-237

45. Rangarajan P, Karthikeyan A, Lu J, Ling EA, Dheen ST (2015) Sirtuin 3 regulates Foxo3a-mediated antioxidant pathway in microglia. Neuroscience 311:398-414. doi:10.1016/j.neu roscience.2015.10.048 
46. Rose G, Dato S, Altomare K, Bellizzi D, Garasto S, Greco V, Passarino G, Feraco E, Mari V, Barbi C, BonaFe M, Franceschi C, Tan Q, Boiko S, Yashin AI, De Benedictis G (2003) Variability of the SIRT3 gene, human silent information regulator Sir2 homologue, and survivorship in the elderly. Exp Gerontol 38:1065-1070. doi:10.1016/S0531-5565(03)00209-2

47. Roth GS, Ingram DK, Lane MA (2001) Caloric restriction in primates and relevance to humans. Ann N Y Acad Sci 928:305-315. doi:10.1111/j.1749-6632.2001.tb05660.x

48. Schächinger V, Britten MB, Zeiher AM (2000) Prognostic impact of coronary vasodilator dysfunction on adverse long-term outcome of coronary heart disease. Circulation 101:1899-1906. doi:10.1161/01.CIR.101.16.1899

49. Someya S, Yu W, Hallows WC, Xu J, Vann JM, Leeuwenburgh C, Tanokura M, Denu JM, Prolla TA (2010) Sirt3 mediates reduction of oxidative damage and prevention of age-related hearing loss under caloric restriction. Cell 143:802-812. doi:10. 1016/j.cell.2010.10.002

50. Speer T, Rohrer L, Blyszczuk P, Shroff R, Kuschnerus K, Kränkel N, Kania G, Zewinger S, Akhmedov A, Shi Y, Martin T, Perisa D, Winnik S, Müller MF, Sester U, Wernicke G, Jung A, Gutteck U, Eriksson U, Geisel J, Deanfield J, von Eckardstein A, Lüscher TF, Fliser D, Bahlmann FH, Landmesser U (2013) Abnormal high-density lipoprotein induces endothelial dysfunction via activation of toll-like receptor-2. Immunity 38:754-768. doi:10.1016/j.immuni.2013.02.009

51. Sundaresan NR, Gupta M, Kim G, Rajamohan SB, Isbatan A, Gupta MP (2009) Sirt3 blocks the cardiac hypertrophic response by augmenting Foxo3a-dependent antioxidant defense mechanisms in mice. J Clin Invest 119:2758-2771. doi:10.1172/ JCI39162

52. Tanner FC, Loo B, Shaw S, Greutert H, Bachschmid MM, Berrozpe M, Rozenberg I, Blau N, Siebenmann R, Schmidli J, Meyer $P$, Luscher TF (2007) Inactivity of nitric oxide synthase gene in the atherosclerotic human carotid artery. Basic Res Cardiol 102:308-317. doi:10.1007/s00395-007-0650-7

53. Tao R, Coleman MC, Pennington JD, Ozden O, Park SH, Jiang H, Kim HS, Flynn CR, Hill S, McDonald WH, Olivier AK, Spitz DR, Gius D (2010) Sirt3-mediated deacetylation of evolutionarily conserved lysine 122 regulates MnSOD activity in response to stress. Mol Cell 40:893-904. doi:10.1016/j.molcel.2010.12.013

54. Tseng AHH, Shieh S-S, Wang DL (2013) SIRT3 deacetylates FOXO3 to protect mitochondria against oxidative damage. Free Radic Biol Med 63:222-234. doi:10.1016/j.freeradbiomed.2013. 05.002

55. Winnik S, Gaul DS, Preitner F, Lohmann C, Weber J, Miranda MX, Liu Y, Tits LJ, Mateos JM, Brokopp CE, Auwerx J, Thorens B, Lüscher TF, Matter CM (2014) Deletion of Sirt3 does not affect atherosclerosis but accelerates weight gain and impairs rapid metabolic adaptation in LDL receptor knockout mice: implications for cardiovascular risk factor development. Basic Res Cardiol 109:399. doi:10.1007/s00395-013-0399-0

56. Winnik S, Lohmann C, Richter EK, Schafer N, Song WL, Leiber F, Mocharla P, Hofmann J, Klingenberg R, Boren J, Becher B, FitzGerald GA, Luscher TF, Matter CM, Beer JH (2011) Dietary alpha-linolenic acid diminishes experimental atherogenesis and restricts T cell-driven inflammation. Eur Heart J 32:2573-2584. doi:10.1093/eurheartj/ehq501

57. Winnik S, Lohmann C, Siciliani G, von Lukowicz T, Kuschnerus K, Kraenkel N, Brokopp CE, Enseleit F, Michels S, Ruschitzka F, Lüscher TF, Matter CM (2013) Systemic VEGF inhibition accelerates experimental atherosclerosis and disrupts endothelial homeostasis-implications for cardiovascular safety. Int J Cardiol 168:2453-2461. doi:10.1016/j.ijcard.2013.03.010

58. Winnik S, Auwerx J, Sinclair DA, Matter CM (2015) Protective effects of sirtuins in cardiovascular diseases: from bench to bedside. Eur Heart J 36:3404-3412. doi:10.1093/eurheartj/ ehv290

59. Yu W, Dittenhafer-Reed KE, Denu JM (2012) SIRT3 protein deacetylates isocitrate dehydrogenase 2 (IDH2) and regulates mitochondrial redox status. J Biol Chem 287:14078-14086. doi:10.1074/jbc.M112.355206

60. Schäfer N, Lohmann C, Winnik S, van Tits LJ, Miranda MX, Vergopoulos A, Ruschitzka F, Nussberger J, Berger S, Lüscher TF, Verrey F, Matter CM (2013) Endothelial mineralocorticoid receptor activation mediates endothelial dysfunction in diet-induced obesity. Eur Heart J 34:3515-3524 